\title{
T7 RNA Polymerase Transcription with 5-position Modified UTP derivatives
}

\author{
Jonathan D. Vaught, Torin Dewey and Bruce E. Eaton*
}

College of Physical and Mathematical Sciences

Department of Chemistry

North Carolina State University

Raleigh, NC 27695

\section{Supplemental Information}

The following tables contain the average relative velocities $\left(\mathrm{V}_{\text {rel }}\right)$ for each UTP derivative 1-7 and UTP obtained from the integrated phosphorimage band intensity of box A-1 for the transcript stopped at position +14 or box A- 2 for transcript extended past +14 as described in the manuscript. The RNA transcripts were labeled by using $\left[\gamma^{32} \mathrm{P}\right]-$ GTP during transcription ensuring that only the 5'-end was labeled. The RNA transcripts were then separated by $18 \%$ denaturing PAGE and the boxed areas A-1 and A-2 used in phosphorimaging to determine the fraction of transcript stopped and extended respectively. The tables are the average values obtained from three kinetic runs for each UTP derivative. For UTP the average values are from 21 kinetic runs. For each UTP derivative and UTP the data are plotted to show the fit to the Michealis-Menton equation as per the manuscript discussion. The $\mathrm{V}_{\text {rel }}$ values for $\mathbf{3}, \mathbf{7}, \mathbf{6}$, and UTP above $100 \mu \mathrm{M}$, and for $\mathbf{1 , 2 , 4}$, and $\mathbf{5}$ above $320 \mu \mathrm{M}$ were not included in the plots. At these higher levels of triphosphate these average $\mathrm{V}_{\text {rel }}$ values were either the same (within 
experimental error) as the upper limit shown in the plots or slightly lower. Omitting these high triphosphate concentrations gave an improved curve fit for UTP and the UTP derivatives.

Triphosphate $1(\mathrm{M})$ avg. $\mathrm{V}_{\text {rel }}$

$\begin{array}{lr}0.000316 & 17.7 \\ 9.98 \mathrm{E}-05 & 12.3 \\ 3.15 \mathrm{E}-05 & 6.5 \\ 9.97 \mathrm{E}-06 & 2.5 \\ 3.15 \mathrm{E}-06 & 0.9 \\ 9.95 \mathrm{E}-07 & 0.3 \\ 3.14 \mathrm{E}-07 & 0.1 \\ 9.93 \mathrm{E}-08 & 0.1\end{array}$

Triphosphate 2(M) avg. $\mathrm{V}_{\text {rel }}$ 1.00E-03 $\quad 12.1$

3.16E-04 $\quad 13.5$

9.98E-05 $\quad 12.0$

3.15E-05 $\quad 7.5$

9.97E-06 2.9

3.15E-06 $\quad 1.0$

9.95E-07 0.3

3.14E-07 0.2

9.93E-08 $\quad 0.0$

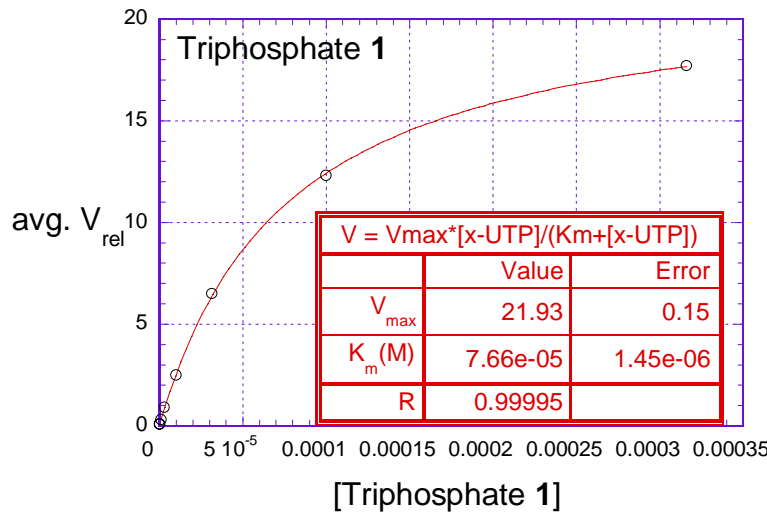

[Triphosphate 1]
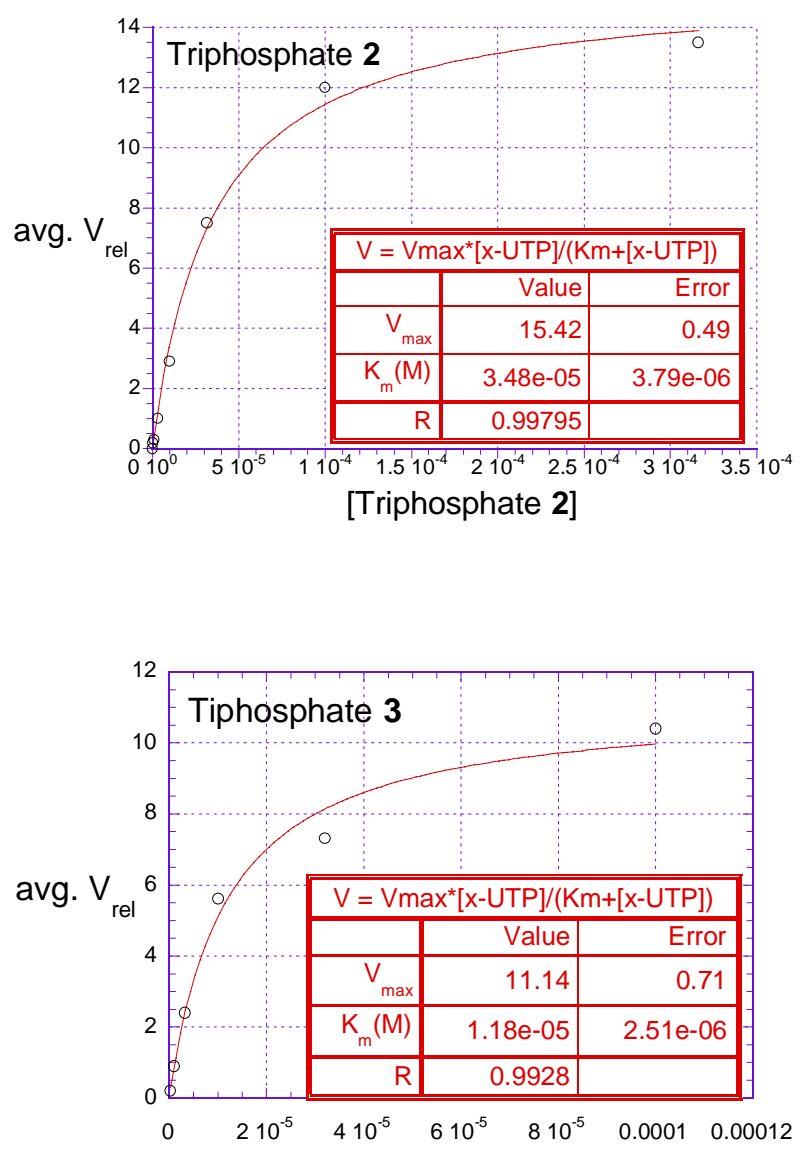

[Tiphosphate 3]
Triphosphate 3(M) avg. $\mathrm{V}_{\text {rel }}$ 3.20E-04 9.8 $1.00 \mathrm{E}-05 \quad 10.4$

3.10E-05 $\quad 7.3$

$1.00 \mathrm{E}-06 \quad 5.6$

3.20E-06 2.4

$1.00 \mathrm{E}-07 \quad 0.9$

3.14E-07 $\quad 0.2$ 
Triphosphate 4(M) avg. $\mathrm{V}_{\text {rel }}$ $0.001 \quad 14.3$ $0.000316 \quad 13.3$

9.98E-05 $\quad 7.8$

3.15E-05 3.2

9.97E-06 1.1

3.15E-06 0.4

$9.95 \mathrm{E}-07 \quad 0.1$

3.14E-07 0.1

9.93E-08 0.1

Triphosphate 5(M) avg. $\mathrm{V}_{\text {rel }}$

$0.001 \quad 17.3$

$0.000316 \quad 20.5$

9.98E-05 $\quad 16.8$

3.15E-05 $\quad 10.4$

9.97E-06 $\quad 4.5$

3.15E-06 $\quad 1.7$

9.95E-07 0.5

3.14E-07 0.2

9.93E-08 0.0

Triphosphate 6(M) avg. $\mathrm{V}_{\text {rel }}$

$1.00 \mathrm{E}-03 \quad 8.7$

3.16E-04 10.8

9.98E-05 11.2

3.15E-05 9.5

9.97E-06 6.6

3.15E-06 3.1

9.95E-07 1.2

3.14E-07 0.4

9.93E-08 0.1
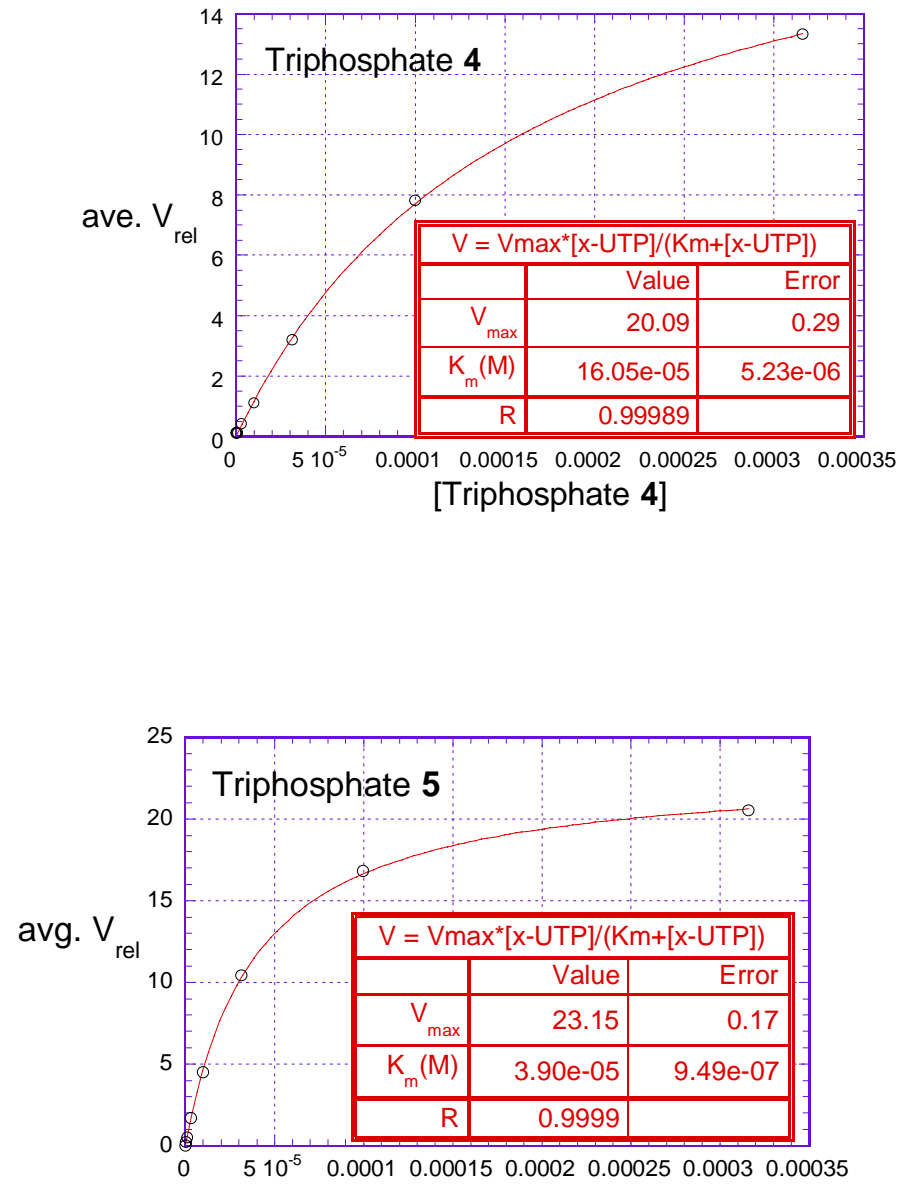

[Triphosphate 5]

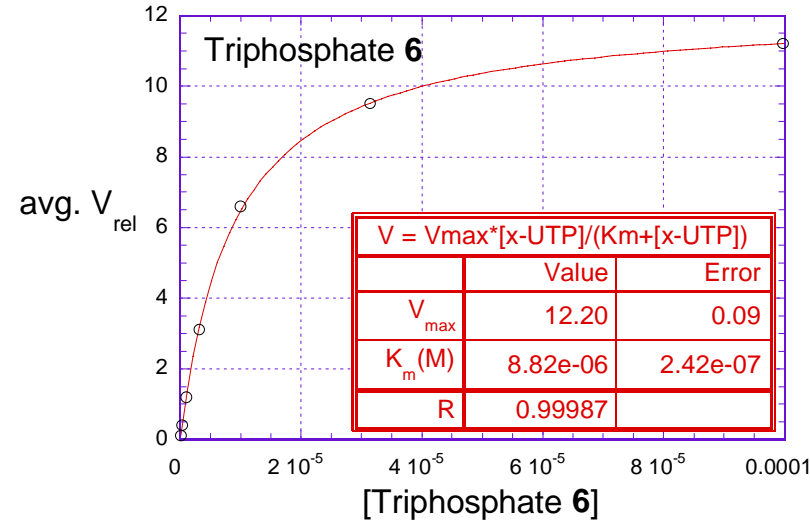




$\begin{array}{rr}\text { Triphosphate } 7(\mathrm{M}) & \text { avg. } \mathrm{V}_{\text {rel }} \\ 3.20 \mathrm{E}-04 & 8.9 \\ 1.00 \mathrm{E}-04 & 9.3 \\ 3.20 \mathrm{E}-05 & 6.9 \\ 1.00 \mathrm{E}-05 & 3.8 \\ 3.20 \mathrm{E}-06 & 1.8 \\ 1.00 \mathrm{E}-06 & 0.6 \\ 3.20 \mathrm{E}-07 & 0.2\end{array}$

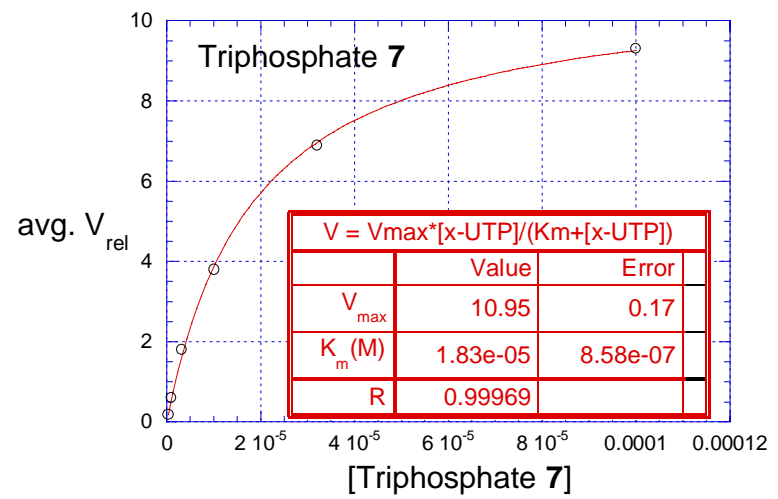

$\begin{array}{lr}\text { UTP(M) avg. } & \mathrm{V}_{\text {rel }} \\ 1.00 \mathrm{E}-03 & 15.0 \\ 1.00 \mathrm{E}-04 & 15.0 \\ 1.00 \mathrm{E}-05 & 6.7 \\ 1.00 \mathrm{E}-06 & 1.0 \\ 1.00 \mathrm{E}-07 & 0.2\end{array}$

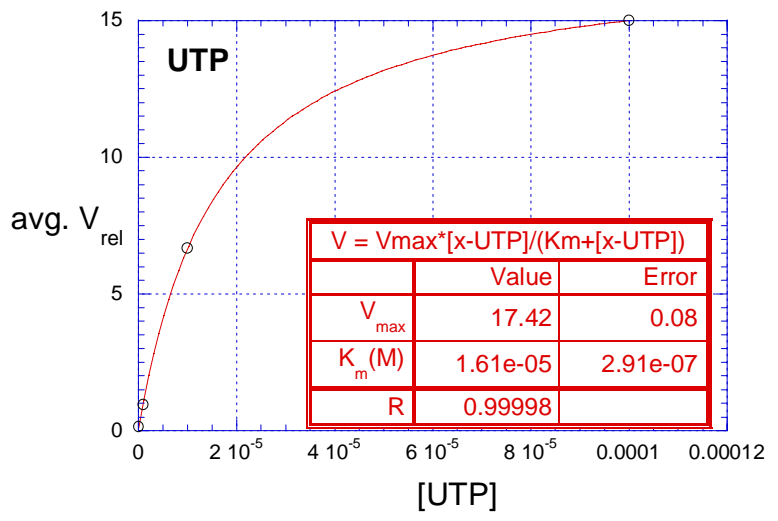

General Experimental. All compounds were prepared from reagent grade starting materials as purchased from Aldrich unless otherwise noted. Tetrakis[Triphenylphosphine]palladium(0) was used as received from Strem Chemicals. 5-Iodo2',3'-isopropylideneuridine was prepared as described previously. ${ }^{24}$ NMR spectra were recorded on Varian 300 or $400 \mathrm{MHz}$ spectrometers and are reported as $\delta$ values, 
referenced to solvent resonances. High resolution mass spectra were recorded at the Mass Spectrometry facility at Duke University(Durham, NC).

Synthesis of nucleoside precursors of triphosphates 1a - 7a. Nucleosides 1a, 2a, and 4a were prepared as previously described. ${ }^{1}$ Nucleosides $\mathbf{3 a}, \mathbf{5 a}, \mathbf{6 a}$, and 7a were prepared by a similar procedure. In a heavy-walled glass vessel $(30 \mathrm{~mL})$ equipped with a Teflon vacuum stopcock was added 5-Iodo-2',3'-isopropylideneuridine, 3 eq. of amine, 5 equiv. triethylamine, 0.1 equiv. tetrakis-[triphenylphosphine]palladium(0), and $5 \mathrm{~mL}$ THF(from MBraun solvent purification system). The vessel was evacuated and filled with 50 psi of $\mathrm{CO}$ three times, then sealed and heated at $70{ }^{\circ} \mathrm{C}$ for $48 \mathrm{hrs}$. The reaction was cooled and carefully vented into a fume hood, and the crude solution applied to a pad of silica $(20 \mathrm{~g})$ in a $150 \mathrm{~mL}$ glass-fritted Buchner funnel $(150 \mathrm{~mL})$. The product was eluted with THF(50 mL) and then dried to a bubbly yellow solid in vacuo. The yellow solid was washed with ether $(50 \mathrm{~mL})$ providing the desired product as a white solid.
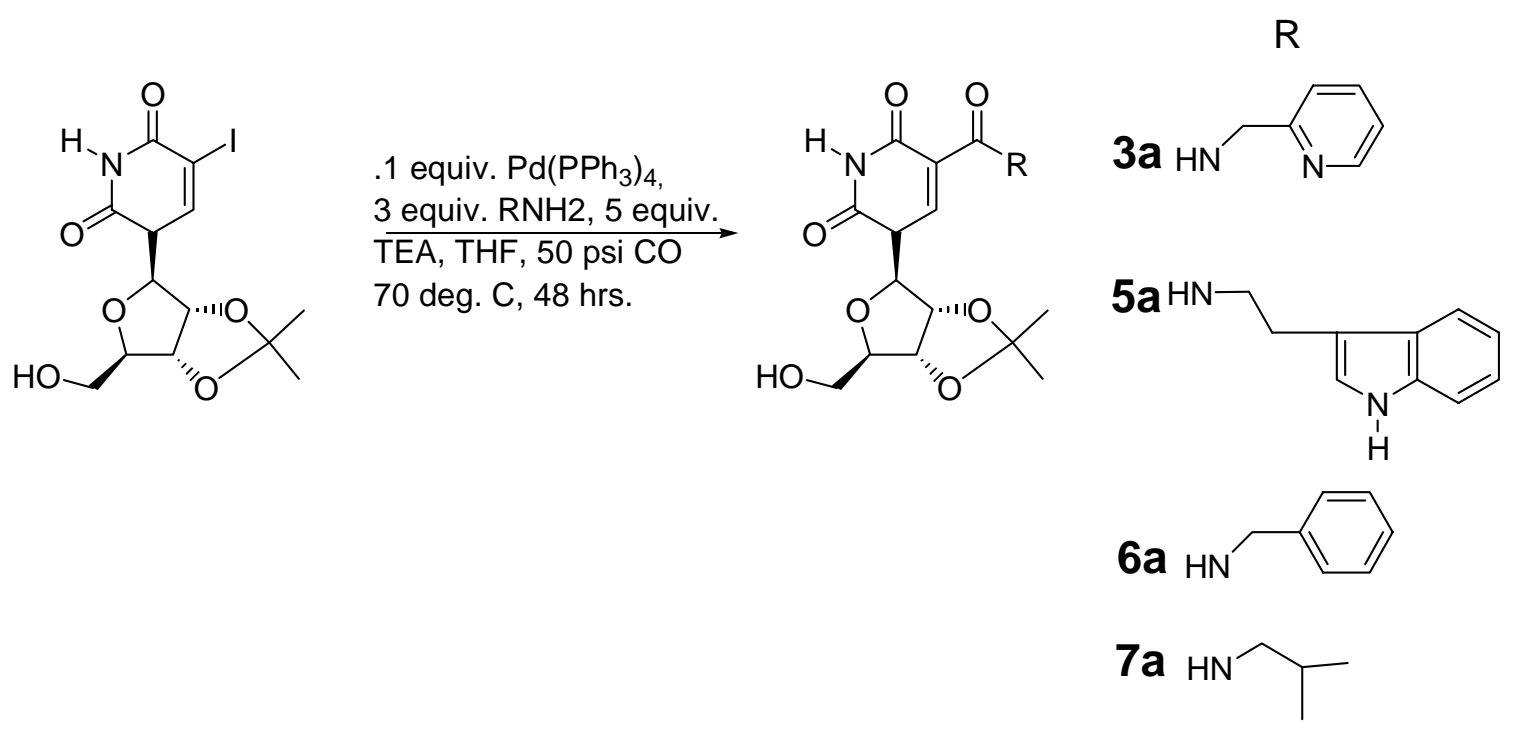

Spectroscopic data for Nucleosides 3a, 5a, 6a, 7a, and Nucleotides 1-7.

3a. ${ }^{1} \mathrm{H}$ NMR (300 MHz, DMSO-d 6 ) $\delta 11.95(\mathrm{~s}, 1 \mathrm{H}), 9.35(\mathrm{t}, J=6 \mathrm{~Hz}, 1 \mathrm{H}), 8.65$

$(\mathrm{s}, 1 \mathrm{H}), 8.50(\mathrm{~d}, J=5 \mathrm{~Hz}, 1 \mathrm{H}), 7.74(\mathrm{t}, J=8 \mathrm{~Hz}, 1 \mathrm{H}), 7.26(\mathrm{~m}, 2 \mathrm{H}), 5.84(\mathrm{~d}, J=2 \mathrm{~Hz}$, 
$1 \mathrm{H}), 5.10(\mathrm{t}, J=5 \mathrm{~Hz}, 1 \mathrm{H}), 4.91(\mathrm{~d}, J=6 \mathrm{~Hz}, 1 \mathrm{H}), 4.73(\mathrm{dd}, J=6,3 \mathrm{~Hz}, 1 \mathrm{H}), 4.56(\mathrm{~d}, J=$ $5 \mathrm{~Hz}, 2 \mathrm{H}), 4.19(\mathrm{~d}, J=4 \mathrm{~Hz}, 1 \mathrm{H}), 3.55(\mathrm{t}, J=4 \mathrm{~Hz}, 2 \mathrm{H}), 1.46(\mathrm{~s}, 3 \mathrm{H}), 1.26(\mathrm{~s}, 3 \mathrm{H}) .{ }^{13} \mathrm{C}$ NMR (300 MHz, DMSO -d6) $\delta 163.9,162.3,158.4,150.2,149.6,148.0,137.5,122.9$, $121.9,113.3,105.6,93.7,88.2,85.2,81.4,61.9,44.8,27.6,25.7$. HRMS (FAB) calcd. for $\mathrm{C}_{19} \mathrm{H}_{23} \mathrm{~N}_{4} \mathrm{O}_{7}(\mathrm{MH}+)$ 419.1566, found 419.1556 .

5a. ${ }^{1} \mathrm{H}$ NMR (400 MHz, DMSO-d $\left.{ }_{6}\right) \delta 11.85(\mathrm{~s}, 1 \mathrm{H}), 10.81(\mathrm{~s}, 1 \mathrm{H}), 8.80(\mathrm{t}, J=6$ Hz, 1H), 8.62 (s, 1H), 7.57 (d, $J=8 \mathrm{~Hz}, 1 \mathrm{H}) 7.33$ (d, $J=8 \mathrm{~Hz}, 1 \mathrm{H}), 7.15$ (s, 1H), 7.05 (t, $J=8 \mathrm{~Hz}, 1 \mathrm{H}), 6.96(\mathrm{t}, J=8 \mathrm{~Hz}, 1 \mathrm{H}), 5.85(\mathrm{~d}, J=2 \mathrm{~Hz}, 1 \mathrm{H}), 5.11(\mathrm{t}, J=4 \mathrm{~Hz}, 1 \mathrm{H}), 4.91$ (dd, $J=6 \mathrm{~Hz}, 2 \mathrm{~Hz}, 1 \mathrm{H}), 4.74$ (dd, $J=6 \mathrm{~Hz}, 3 \mathrm{~Hz}, 1 \mathrm{H}), 3.55$ (m, 4H), 2.89 (t, $J=7 \mathrm{~Hz}$, 2H), 1.47 (s, 3H), 1.27 (s, 3H). ${ }^{13} \mathrm{C}$ NMR (400 MHz, DMSO-d 6 ) $\delta 163.9,162.1,150.2$, $147.7,137.0,127.8,123.5,121.7,119.0,118.9,113.3,112.2,112.1,105.7,93.6,88.1$, 85.1, 81.4, 61.9, 27.6, 25.9, 25.8; HRMS (FAB) calcd. for $\mathrm{C}_{23} \mathrm{H}_{26} \mathrm{O}_{7} \mathrm{~N}_{4}(\mathrm{MH}+)$ 470.1801, found 470.1802 .

6a. ${ }^{1} \mathrm{H}$ NMR (300 MHz, DMSO-d 6 ) $\delta 11.94$ (s, 1H), 9.09 (t, $\left.J=6 \mathrm{~Hz}, 1 \mathrm{H}\right), 8.66$ (s, 1H), $7.27(\mathrm{~m}, 5 \mathrm{H}), 5.86(\mathrm{~s}, 1 \mathrm{H}), 5.10(\mathrm{t}, J=4 \mathrm{~Hz}, 1 \mathrm{H}), 4.92(\mathrm{~d}, J=7 \mathrm{~Hz}, 1 \mathrm{H}), 4.74$ (dd, $J=7,3 \mathrm{~Hz}, 1 \mathrm{H}), 4.47$ (d, $J=6 \mathrm{~Hz}, 2 \mathrm{H}), 4.22(\mathrm{~d}, J=6 \mathrm{~Hz}, 1 \mathrm{H}), 3.57$ (t, $J=4 \mathrm{~Hz}$, 2H), 1.47 (s, 3H), 1.27 (s, 3H). ${ }^{13} \mathrm{C}$ NMR (400 MHz, DMSO-d $\left.\mathrm{d}_{6}\right) \delta$ 164.0, 162.3, 150.2, $148.0,141.6,139.9,129.1,128.0,127.6,113.3,105.6,93.6,88.2,85.2,81.4,61.9,43.7$, 42.8, 27.6, 25.7. HRMS (FAB) calcd. for $\mathrm{C}_{20} \mathrm{H}_{24} \mathrm{~N}_{3} \mathrm{O}_{7}(\mathrm{MH}+)$ 418.1614, found 418.1595.

7a. ${ }^{1} \mathrm{H}$ NMR (300 MHz, DMSO-d 6 ) $\delta 11.92,(\mathrm{~s}, 1 \mathrm{H}), 8.75(\mathrm{t}, J=6 \mathrm{~Hz}, 1 \mathrm{H}), 8.60$ (s, $1 \mathrm{H}), 5.83(\mathrm{~d}, J=2 \mathrm{~Hz}, 1 \mathrm{H}), 5.09(\mathrm{t}, J=5 \mathrm{~Hz}, 1 \mathrm{H}), 4.91(\mathrm{dd}, J=6,2 \mathrm{~Hz}, 1 \mathrm{H}), 4.73(\mathrm{dd}, J$ $=6,3 \mathrm{~Hz}, 1 \mathrm{H}), 4.18(\mathrm{dd}, J=7,4 \mathrm{~Hz}, 1 \mathrm{H}), 3.55(\mathrm{t}, J=4 \mathrm{~Hz}, 2 \mathrm{H}), 3.08(\mathrm{t}, J=6 \mathrm{~Hz}, 2 \mathrm{H})$, $1.73(\mathrm{~m}, 1 \mathrm{H}), 1.46(\mathrm{~s}, 3 \mathrm{H}), 1.26(\mathrm{~s}, 3 \mathrm{H}), 0.85(\mathrm{~d}, J=7 \mathrm{~Hz}, 6 \mathrm{H}) .{ }^{13} \mathrm{C} \mathrm{NMR}(400 \mathrm{MHz}$, 
DMSO-d $\left._{6}\right) \delta 164.2,162.1,150.2,147.7,113.3,105.7,93.6,88.1,85.1,81.4,61.9,46.4$, 28.8, 27.6, 25.7, 20.7. HRMS (FAB) calcd. for $\mathrm{C}_{17} \mathrm{H}_{26} \mathrm{~N}_{3} \mathrm{O}_{7}(\mathrm{MH}+)$ 384.1770, found 384.1783 .

Synthesis of UTP Derivatives 1 - 7. All 5-position modified UTP derivatives were prepared as described by the method of Ekstein. ${ }^{2}$

1. UV $\lambda_{\max } 275 \mathrm{~nm} \varepsilon 12,900$. HRMS (FAB) calcd. for $\mathrm{C}_{12} \mathrm{H}_{22} \mathrm{O}_{16} \mathrm{~N}_{4} \mathrm{P}_{3}(\mathrm{MH}+)$ 571.0243, found 571.0251 .

2. UV $\lambda_{\max } 276 \mathrm{~nm} \varepsilon 13,400$. HRMS (FAB) calcd. for $\mathrm{C}_{16} \mathrm{H}_{22} \mathrm{O}_{16} \mathrm{~N}_{4} \mathrm{P}_{3}(\mathrm{MH}+)$ 619.0225, found 619.0193 .

3. UV $\lambda_{\max } 269 \mathrm{~nm} \varepsilon$ 17,900. HRMS (FAB) calcd. for $\mathrm{C}_{16} \mathrm{H}_{22} \mathrm{O}_{16} \mathrm{~N}_{4} \mathrm{P}_{3}(\mathrm{MH}+)$ 619.0225, found 619.0234 .

4. UV $\lambda_{\max } 276 \mathrm{~nm} \varepsilon 12,600$. HRMS (FAB) calcd. for $\mathrm{C}_{15} \mathrm{H}_{23} \mathrm{O}_{16} \mathrm{~N}_{5} \mathrm{P}_{3}(\mathrm{MH}+)$ 622.0353, found 622.0330 .

5. UV $\lambda_{\max } 278 \mathrm{~nm} \varepsilon 20,000$. HRMS (FAB) calcd. for $\mathrm{C}_{20} \mathrm{H}_{26} \mathrm{O}_{16} \mathrm{~N}_{4} \mathrm{P}_{3}(\mathrm{MH}+)$ 671.1451, found 671.1442 .

6. UV $\lambda_{\max } 276 \mathrm{~nm} \varepsilon 16,000$. HRMS (FAB) calcd. for $\mathrm{C}_{23} \mathrm{H}_{38} \mathrm{O}_{16} \mathrm{~N}_{4} \mathrm{P}_{3}\left(\mathrm{MC}_{6} \mathrm{H}_{17} \mathrm{~N}+\right)$ 719.1494 , found 719.1464 .

7. UV $\lambda_{\max } 276 \mathrm{~nm} \varepsilon 12,400$. HRMS (FAB) calcd. for $\mathrm{C}_{20} \mathrm{H}_{40} \mathrm{O}_{16} \mathrm{~N}_{4} \mathrm{P}_{3}\left(\mathrm{MC}_{6} \mathrm{H}_{17} \mathrm{~N}+\right)$ 685.1652 , found 685.1629 . 


\section{References}

(1) (a) Dewey, T. M.; Zyzniewski, M. C.; Eaton, B. E. Nucleosides \& Nucleotides 1996, 15, 1611-1617. (b) Dewey, T. M.; Mundt, A.; Crouch, G.J.; Zyzniewski, M. C.; Eaton, B.E. J. Am. Chem. Soc. 1995, 1178474.

(2) Ludwig, J.; Eckstein, F. J. Org. Chem. 1989, 54, 631-635. 